\title{
Can metronomic chemotherapy be an alternative to sorafenib in advanced hepatocellular carcinoma?
}

\author{
Do Young Kim \\ Department of Internal Medicine, Yonsei University College of Medicine, Seoul, Korea
}

Keywords: Hepatocellular carcinoma; Sorafenib; Hepatic arterial infusion chemotherapy; Metronomic chemotherapy

\section{See Article on Page 128}

Conventional hepatic arterial infusion chemotherapy (HAIC), consisting of 5-fluorouracil (5-FU) and cisplatin delivered via implanted chemoport every 4 weeks, is a form of regional to systemic chemotherapy to treat unresectable or advanced hepatocellular carcinoma (HCC). The idea of localized infusion of cytotoxic agents into liver originated from hepatic metastasis of colorectal cancer.' It is interesting that the first application of HAIC to HCC has been done in Western country, ${ }^{2,3}$ even though that modality is now being used in Japan, Korea and Taiwan. In a Japanese retrospective study of 48 patients with portal vein tumor thrombosis, the objective (complete and partial) response rates were high (48\%) and 3-year survival rates were $25 \%{ }^{4}$ In a large Japanese cohort, the median survival times of patients who underwent HAIC was 14.0 months, which were significantly higher than in those who did not receive active treatment $(5.2$ months, $P<0.0001) .{ }^{5}$ In contrast with daily, low doses of cisplatin $\left(7 \mathrm{mg} / \mathrm{m}^{2}\right.$ on day $\left.1-5\right)$ and $5-\mathrm{FU}(170$ $\mathrm{mg} / \mathrm{m}^{2}$ on day $\left.1-5\right)$ in Japanese practice, Korean regimen consisted of higher cisplatin $\left(60 \mathrm{mg} / \mathrm{m}^{2}\right.$ on day 2) and $5-\mathrm{FU}\left(500 \mathrm{mg} / \mathrm{m}^{2}\right.$ on day $1-3$ ) doses repeated every 4 weeks. ${ }^{6,7}$ The dictionary meaning of 'metronome' is a device that produces regular, metrical ticks or beats; these represent a fixed, regular pulse. In the current issue by Yang et al. ${ }^{8}, 1$ cycle of metronomic chemotherapy (MET) was composed of single low doses of cisplatin $\left(15 \mathrm{mg} / \mathrm{m}^{2}\right)$ and 5 -FU $\left(50 \mathrm{mg} / \mathrm{m}^{2}\right)$ infused via hepatic arterial infusion chemoport for 3 weeks (1 week break). ${ }^{8}$ The authors aimed to retrospectively compare the efficacy and safety between MET and sorafenib in patients with advanced (BCLC-C) stage. In 54 and 53 patients who received MET and sorafenib treatment, the median overall survival (OS) was 158 and 117 days, respectively $(P=0.029)$. The disease control rate assessed at week 8 tended was higher in MET group than in sroafenib group $(53.7 \%$ vs. $22.0 \% ; P=0.014)$. In subgroup of patients with Child-Pugh class B, the median OS was significantly longer in MET group than in sorafenib group (190 vs. 58 days, $P<0.001)$. In terms of safety, although there is no information on the proportion of treatment discontinuation due to adverse events (AEs), MET was more related to hematologic AE including leukopenia and thrombocytopenia, whereas the majority of sorafenib-related toxicity was hand foot skin reaction and $A E$ of gastrointestinal tract. Based on these results, the authors state that MET would be an alternative to sorafenib in HCC patients with BCLC-C stage, particularly if they don't have preserved liver function.

This article by Yang et al. ${ }^{8}$ suggests that MET, a sort of regional

\section{Abbreviations:}

5-FU, 5-fluorouracil; AE, adverse events; BCLC-C, Barcelona Clinic Liver Cancer-C; HAIC, hepatic arterial infusion chemotherapy; HCC, hepatocellular carcinoma; MET, metronomic chemotherapy; OS, overall survival

\section{Corresponding author : Do Young Kim}

Department of Internal Medicine, Yonsei University College of Medicine, 50-1 Yonsei-ro, Seodaemun-gu, Seoul 03722, Korea

Tel: +82-2-2228-1992, Fax: +82-2-393-6884

E-mail:dyk1025@yuhs.ac

http://orcid.org/0000-0002-8327-3439 
cytotoxic chemotherapy, might be a potential option for advanced HCC. The MET seems to have an advantage of lower rates of toxicity such as hepatic decompensation compared with conventional HAIC. In addition, there seems to be no need of hospitalization for drug administration. However, the study has several limitations. Apart from the retrospective nature of data, sample size is not enough to draw the conclusion that MET is comparable to sorafenib. Most importantly, the variables are imbalanced between the two groups. Although there was no significant statistical difference, more patients in sorafenib group had main portal vein tumor thrombosis than in MET group (45.3\% vs. 33.3\%). The frequency of extrahepatic metastasis, which is an important prognostic factor, was also higher in sorafenib group than in MET group $(66.0 \%$ vs. $51.9 \%, P=0.136)$.

Though the role and positioning of HAIC, including MET, has not yet been established in $\mathrm{HCC}$, a recently published paper reporting the efficacy of sorafenib plus HAIC suggests a possibility of combining systemic therapy and regional cytotoxic chemotherapy to enhance anti-tumor effect. In the Japanese prospective, multicenter trial, a total 108 patients with advanced HCC were randomized to sorafenib group and sorafenib plus HAIC group (infusion of only cisplatin). The median survival times were 8.7 and 10.6 months, respectively $(P=0.031)$. The response rate was $7.3 \%$ in sorafenib group and $21.7 \%$ in combination group. ${ }^{9}$

For HAIC to be widely accepted as a modality for HCC, more scientific evidences should be accumulated. The deterioration of liver function encountered with repeated cycles of HAIC is a major concern. In this regards, lower probability of liver toxicity by MET is obviously a merit, if we can guarantee that the efficacy of MET is similar to conventional HAIC. With few available regimens for patients with sorafenib failure except regorafenib, HAIC including MET also needs to be further evaluated as a second-line modality ${ }^{10,11}$

\section{Conflicts of Interest}

The author has no conflicts to disclose.

\section{REFERENCES}

1. Sullivan RD, Norcross JW, Watkins E Jr. Chemotherapy of metastatic liver cancer by prolonged hepatic artery infusion. N Engl J Med 1964;270:321-327.

2. Gorgun B, Watne AL. Infusion chemotherapy in hepatoma and metastatic liver tumors. Am J Surg 1967;113:363-368.

3. Patt YZ, Charnsangavei C, Yoffe B, Smith R, Lawrence D, Chuang V, et al. Hepatic arterial infusion of floxuridine, leucovorine, doxorubicin, and cisplatin for hepatocellular carcinoma: effect of hepatitis $B$ and $C$ viral infection on drug toxicity and patient survival. J Clin Oncol 1994;12:1204-1211.

4. Ando E, Tanaka M, Yamashita F, Kuromatsu R, Yutani S, Fukumori K, et al. Hepatic arterial infusion chemotherapy for advanced hepatocellular carcinoma with portal vein tumor thrombosis: analysis of 48 cases. Cancer 2002;95:588-595.

5. Nouso K, Miyahara K, Uchida D, Kuwaki K, Izumi N, Omata M, et al. Effect of hepatic arterial infusion chemotherapy of 5 -fluorouracil and cisplatin for advanced hepatocellular carcinoma in the Nationwide Survey of Primary Liver Cancer in Japan. Br J Cancer 2016;109:19041907.

6. Park JY, Ahn SH, Yoon YJ, Kim JK, Lee HW, Lee DY, et al. Repetitive short-course hepatic arterial infusion chemotherapy with high-dose 5-fluorouracil and cisplatin in patients with advanced hepatocellular carcinoma. Cancer 2007;110:129-137.

7. Woo HY, Bae SH, Park JY, Han KH, Chun HJ, Choi BG, et al. A randomized comparative study of high-dose and low-dose hepatic arterial infusion chemotherapy for intractable, advanced hepatocellular carcinoma. Cancer Chemother Pharmacol 2010;65:373-382.

8. Yang H, Woo HY, Lee SK, Han JW, Jang BH, Nam HC, et al. A comparative study of sorafenib and metronomic chemotherapy for Barcelona Clinic Liver Cancer-stage C hepatocellular carcinoma with poor liver function. Clin Mol Hepatol 2017;23:128-137.

9. Ikeda M, Shimizu S, Sato M, Morimoto M, Kojima Y, Inaba Y, et al. Sorafenib plus hepatic arterial infusion chemotherapy with cisplatin versus sorafenib for advanced hepatocellular carcinoma: randomized phase II trial. Ann Oncol 2016;27:2090-2096.

10. Bruix J, Qin S, Merie P, Granito A, Huang YH, Bodoky G, et al. Regorafenib for patients with hepatocellular carcinoma who progressed on sorafenib treatment (RESORCE): a randomized, double-blind, placebo-controlled, phase 3 trial. Lancet 2017;389:56-66.

11. Shao YY, Liang PC, Wu YM, Huang CC, Huang KW, Cheng JC, et al. A pilot study of hepatic arterial infusion of chemotherapy for patients with advanced hepatocellular carcinoma who have failed antiangiogenic therapy. Liver Int 2013;33:1413-1419. 